\title{
Design of Wireless Sensing Virtual Cloud Desktop System Based on ARM-Linux
}

\author{
https://doi.org/10.3991/ijoe.v13i10.7743 \\ Chunqing Han $\left.{ }^{\square}\right)$, Lin Li \\ Binzhou Tobacco Monopoly Bureau, Shandong, China \\ hanchunqing $0807 @ 163 . \mathrm{com}$
}

\begin{abstract}
Aiming at exploring the wireless sensing network with mass processing in data collection, an embedded wireless sensor system based on ARM-Linux is put forward and designed. The whole system is mainly divided into wireless ZigBee sensing node group part, ARM data processing part, and virtual cloud desktop terminal part. Users' terminal can be connected to the cloud system server through wired or wireless ways. And it is possible to view user system data and wireless terminal data by logging in to an individual account. The results showed that the system can be applied in a lot of fields, such as intelligent transportation, health care, smart home and so on. Based on the above findings, it is concluded that it is important for information aggregation and remote fast monitoring.
\end{abstract}

Keywords-ARM-Linux, wireless sensing, cloud desktop

\section{Introduction}

Wireless sensor network (WSN) is a new network which can sense and collect information by itself. It consists of a large number of wireless sensors in short distance or at long distance. In this system, each sensor node has one or more sensors, which can be used to easily process and calculate these data. Interworking, collection, and processing of information between nodes can be achieved through the established reliable network protocol, so the system can complete the acquisition and real-time processing of a variety of monitoring object information. And these information can be transmitted to the end users through wireless or wired mode, so as to realize the communication between things and human society.

In recent years, wireless sensor networks have become the forefront of scientific research hot-spots, and are paid close attention to by domestic and international researchers and companies. It combines embedded processing technology, short distance wireless communication technology and low power sensing technology. It is a multi-disciplinary, widely cross and promising research direction. Cloud computing is a way of providing ancillary resources to the user in the form of an application service. At present, almost all of the resources can be stored in the cloud, to provide similar cloud services, such as storage cloud, network cloud, cloud, virtual machine cloud, application cloud and so on. The system combines the current internet of things 
technology, embedded technology, and cloud computing technology. It takes the wireless sensor network that is widely used as the wireless terminal, embedded nature as the data core control part, the cloud group server architecture as the data center for processing and carrying large quantities of data, and small-scale embedded devices or common ones as the user terminal.

In wireless sensor networks, ZigBee protocol is one of the implementation methods, which makes the network more intelligent and efficient. As a low-power, reliable and low-cost wireless communication technology, ZigBee has realized some requirements of wireless sensor networks, such as dense distribution, intelligent perception, resistance to interference and so on. This paper studies the principle of ZigBee technology, and on this basis, with IEEE as the based standards, specific analysis is made from the MAC layer, PHY layer to the application layer. As a result, the entire structure is more clearly displayed. In addition, because the terminal of wireless sensor network usually adopts the processor architecture with weak processing performance, the computing power is not too strong, while the ARM architecture chip has a strong processing and computing power. At the same time, Linux, as an open source powerful operating system, coupled with ARM processor, constitutes a whole set of practical and efficient system level application solutions. This paper focuses on the construction of embedded Linux system environment, the construction of UBoot, the kernel transplantation, and the serial port and Socket communication.

\section{Overview of related theories}

\subsection{Basic architecture of wireless sensor network}

Wireless sensor network is mainly composed of three parts: data acquisition part, interactive communication part and core control center. The key part of the wireless sensor network is to integrate the nodes with sensors, data processing unit and communication module for sensor network node to form the sensor network in selforganized way. And they are randomly distributed inside or around the area to be observed [1]. Each node will transmit the data collected to other nodes through the preliminary processing and optimization by wireless means. And then each node will be transmitted in the way of hopping. The monitoring data can be dealt with and transmitted by a number of nodes in the transmission process. And finally, through multistage transmission, the collected information is sent to the network coordinator, and then sent to the central core management node. In this way, a series of information collection and transmission have been completed. The structure of the wireless sensor network is shown in figure 1.

Each white circle in the diagram represents the terminal node, which usually comes with a miniature embedded chip, and the chip will have a data acquisition interface that can sense and retrieve the surrounding information. Terminal nodes, in general, have no very powerful processing capacity, storage space is relatively small, and communication range is relatively narrow. However, its power consumption is generally low, and a section of ordinary dry battery can supply power for a long time. The 
black is the route node, and from the entire network composition functions, each sensor node will take up the two functions of terminal equipment and routing equipment. This period may be a routing node, and the next stage may be taken as a terminal node to obtain the data. Therefore, in addition to its own data collection, it also stores and forwards data sent by other terminals or routing nodes, and completes a series of specific tasks [2].

The ZigBee is selected here and ZigBee is a low power and low cost protocol standard in wireless sensor network technology. The sensor network constructed with ZigBee has the advantages of convenient networking, safety and strong expandability. ZigBee network structure is designed based on standard knife network interconnection reference model. It includes five layers, which are the physical layer, medium access control layer, network layer, application layer and security service providing layer, respectively, as shown in figure 2. The protocol standards of physical layer and MAC layer are defined by the IEEE standard, and the ZigBee alliance is used directly. The network layer, the application layer and the security service provider layer are defined by the ZigBee alliance standard. The application layer consists of application support sub-layers (APS), and ZigBee device objects are application objects that are customized by the user or developer. Each layer can provide services to its upper layer in the form of service access points, and the implementation provided is made by defining a series of basic service instructions (primitives).

In general, the protocol standard of the user's network application layer ZigBee is simple, and specific applications can be implemented. In consequence, for the users, the benefit is: it does not need to implement the entire protocol standard, but only needs smaller memory and code for the realization of protocol specification to meet application requirements. In this way, it can reduce the volume of development, reduce capital consumption, and faster launch their own products.

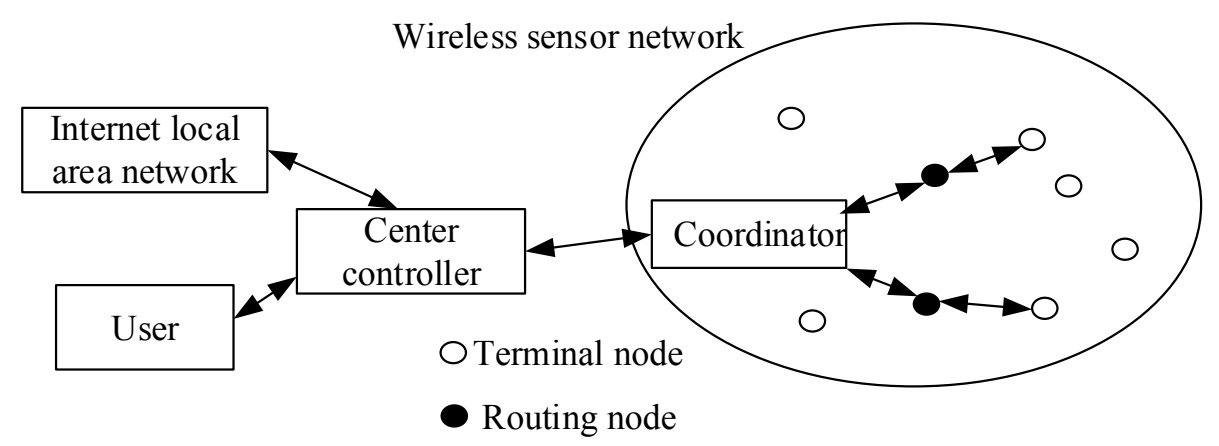

Fig. 1. Structure of wireless sensor network 


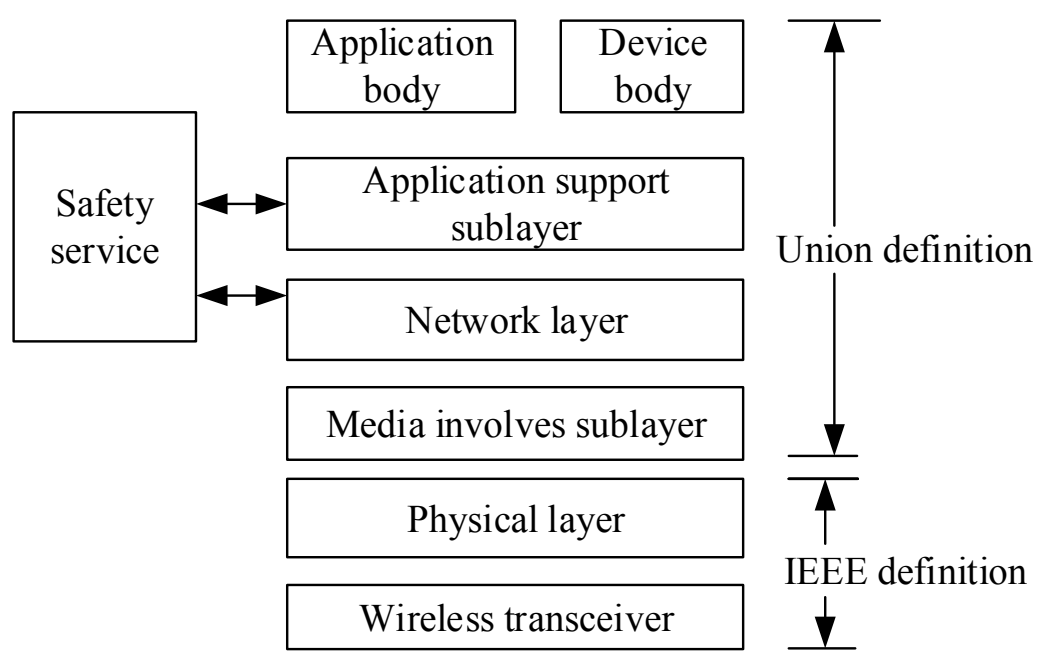

Fig. 2. ZigBee network protocol layers

\subsection{Cloud computing and virtual technology}

Cloud computing is a way of providing ancillary resources to the user in the form of an application service. Today, almost all of the resources can be "cloud", to provide similar cloud services, such as storage cloud, network cloud, cloud, virtual machine cloud, application cloud and so on. Cloud computing requires strong technology to support; otherwise it is difficult to achieve [3]. At present, there are mainly five categories of technologies to provide the support, as shown in figure 3 .

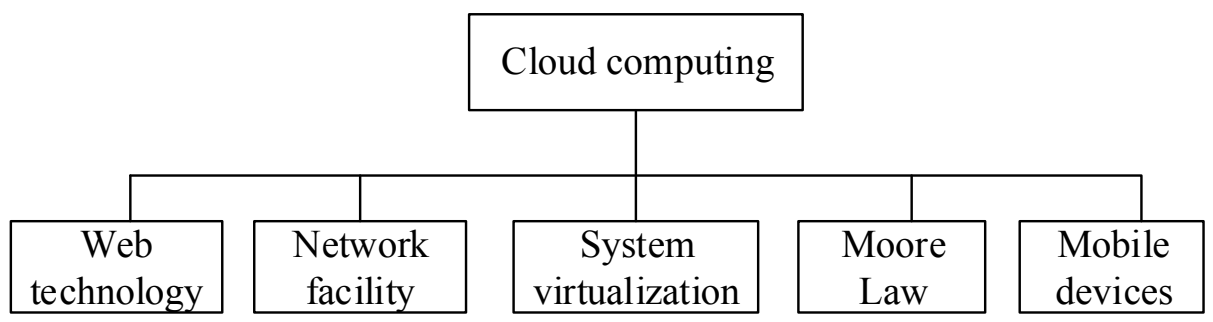

Fig. 3. The technological supporting system of cloud computing

Web technology provides a browser access method, and network infrastructure enhances the performance of cloud computing in bandwidth. The system virtualization is the basis for cloud computing's intelligent operation, Moore's principle provides a theoretical basis for the cloud computing, and mobile device provides more convenient means for accessing to cloud computing. Virtualization technology is a key link in cloud computing that it can be said that without virtualization, there will be no rapid development of cloud computing [4]. At present, virtualization technology has emerged a lot of key technologies: server virtualization, storage virtualization, desk- 
top virtualization, network equipment virtualization, applications virtualization and so on. Among them, the desktop virtualization technology can make that the users do not have to stick to the existing hardware entity. Instead, they can access to the system on the remote server at any time and place through any devices (such as a client phone, pad, and mobile phone) [5].

\section{Method}

\subsection{Overall architecture of wireless sensor network and virtual cloud desktop}

The whole system is mainly divided into wireless ZigBee sensor node group part, ARM data processing part, and virtual cloud desktop terminal part. The wireless sensor node group is mainly composed of wireless communication modules, the ARM data processing part is mainly composed of ARM-Linux embedded system, and the virtual cloud desktop terminal part is mainly composed of virtual cloud desktop client. The overall block diagram of the system is shown in figure 4.

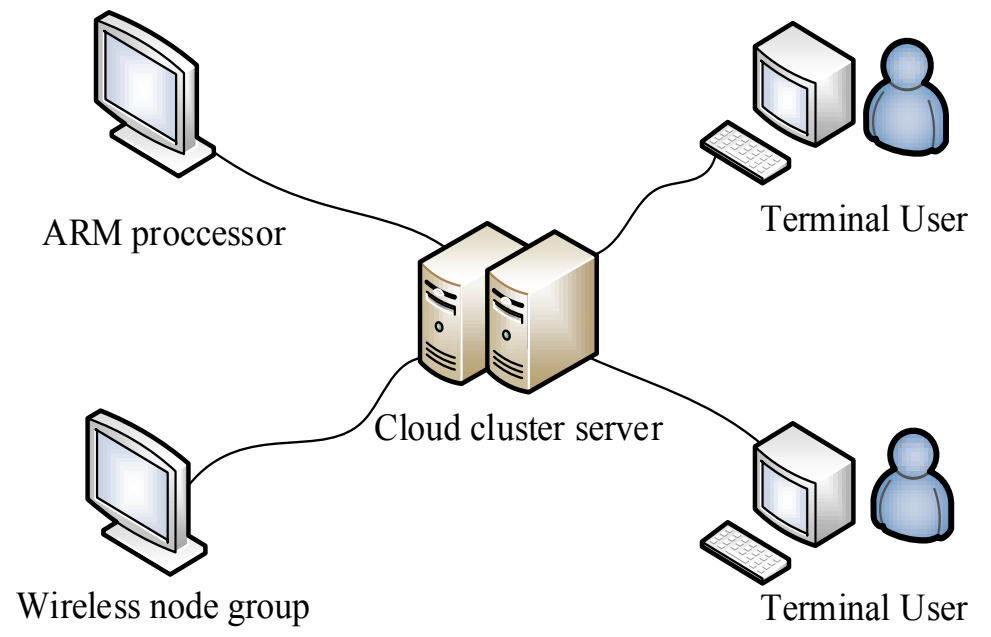

Fig. 4. The overall framework of sensor node hardware system

The left is the ZigBee wireless node group, which can be networked to form a huge sensor node. Then, it is the ARM processor core control section, which is responsible for processing data from the wireless sensor network and data interaction with the cloud server. The cloud swarm server is the data center of the whole system, responsible for data up-link and down-link, and it is also responsible for the computing and control capabilities of the overall system. The rightmost side is the terminal user, who is the user and beneficiary of the final data. It can be remotely connected to the server side on the server to view the appropriate data. 
Specific to the whole software and hardware architecture design, wireless protocol is used for the communication among the node groups, and the coordinator module and the ARM processor part use serial ports for data communication. The system adopts Ethernet mode and cluster server to communicate with each other. The cloud swarm server can consider using IBM or Vmware scheme, but the cost is higher. In order to simulate clustered cloud servers, the system uses common PC to install multiple virtual machines to complete. Virtual cloud desktop terminals and cloud clustered servers can communicate through Ethernet or the wireless network card. For a communication line, the deployment of the system is shown in figure 5:

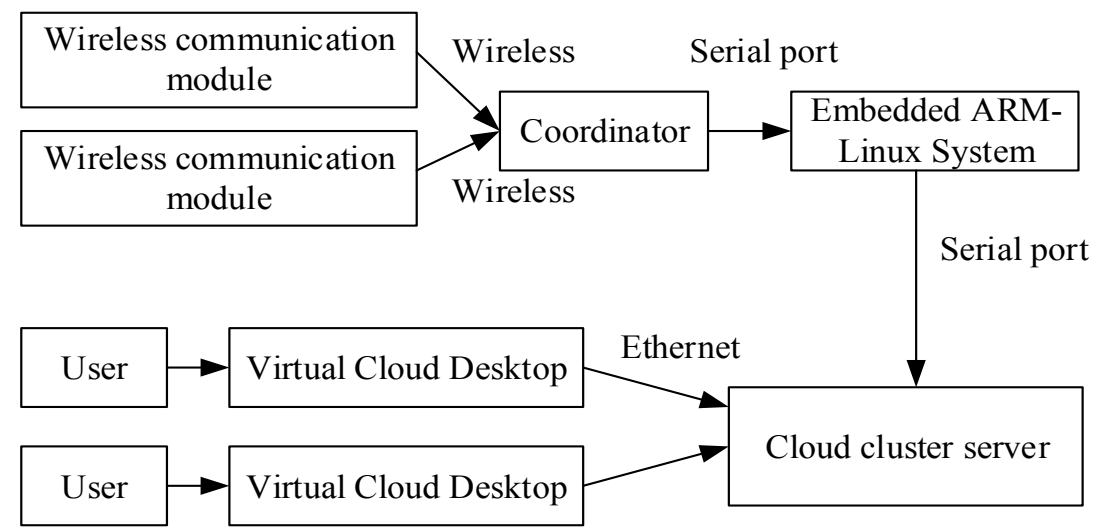

Fig. 5. The system deployment diagram

\subsection{Brief introduction to system hardware platform}

Part of the wireless module of wireless sensor network is the line design and development, using TiCC2430 chip, and PCB micro antenna design. The module size is small, and the data transmission effect is good. ARM data processing uses Mini2440 embedded development board set produced by FriendlyARM company. Mini2440 and wireless sensor network communication is completed through the serial port. It comes with 3 serial ports, UART0, UART1 and UART2, respectively, which in the board, corresponds to CON1, CON2 and CON3, respectively, and has introduced UART0 so that it can use the direct line to connect with the host computer. UART1 and UART2 are not extracted. When needed to be used, it is possible to use wire lead and external device communication.

The development board supports running Linux2.6.32.2, Qtopia-2.2.0, and WindowsCE. Since the Linux system is open source, the system uses the Linux system. The cloud swarm server uses a powerful Intel i5, whose memory is 4G. In order to simulate server virtualization, the virtual machine software VMware was installed. At the same time, a Windows7 systems and a Windows XP system were cloned in order to test system supporting degree. 


\subsection{Data format design of wireless transmission system}

In order to facilitate the interaction of data between modules, communication within the system adopts a specified format. Adopting a unified format facilitates standardization of programming interfaces. The design of the system data format is shown in figure 6:

\begin{tabular}{|l|l|c|c|c|c|}
\hline Data type & Length & $\begin{array}{c}\text { Source } \\
\text { address }\end{array}$ & $\begin{array}{c}\text { Destination } \\
\text { address }\end{array}$ & Parameter & Checksum \\
\hline
\end{tabular}

Fig. 6. Data system format diagram

The data types can distinguish the category that the sending data belongs to, used to distinguish the functions of data. The length is the number of words shown in the diagram, the source address is the sender address of the data, and the destination address is the address to which the data is sent. The parameter $1-\mathrm{N}$ is the parameter carried in the data for the receiver to parse the required information. Check-sum is to carry out the parity check of all data in front.

\section{$4 \quad$ Result and discussion}

Desktop virtualization is a very important part of cloud computing virtualization technology. Desktop virtualization allows the users' desktop environment to be separated from devices equipped with user desktops. The user's system and file are not stored locally but stored on the server to store the user's entire desktop environment. In modern enterprises, it is a very important job for the operation department to install, configure and maintain the desktop system. For each new employee, the operation department needs to be responsible for the system and other auxiliary work, which caused a lot of pressure. At the same time, the relevant data and information security cannot be well monitored, which makes the whole operation cost very high. Through the desktop virtual management, the operation department can configure or manage the system for employees or users only in the data center, thus reducing the unnecessary field operations. In the meanwhile, it is conducive for users to install the software and install system patches. Businesses can dispense the use of expensive terminal desktops, but use lower priced terminal devices to meet the needs of their employees. Only one client can connect to the system on the server to meet the office and other needs. Users can also use more convenient terminal devices, and as long as it has the display function can it meet the needs, such as personal PC, tablet PCs, smart phones and so on.

The whole software is organized by software modules, and each module implements independent functions, so as not to be coupled with other modules as far as possible. This is conducive to later maintenance and updating. All modules of the software are shown in table 1. 
Table 1. Composition of software system of wireless sensor network node

\begin{tabular}{|l|l|l|}
\hline \multicolumn{1}{|c|}{ Name of modules } & \multicolumn{1}{c|}{ Introduction to functions } & \multicolumn{1}{c|}{ Notice } \\
\hline Configured information reading module & To configure information reading & Readconf.c \\
\hline Interface display module & To display the interface controls & Rdpmain.c \\
\hline Log-in module & To log in rdpserver & Login.c \\
\hline Command interaction module & To apply for instructions for field control server & Command.c \\
\hline
\end{tabular}

The configuration information reading module takes on the function of saving user's information and reading user's information. The configuration information is saved in the config.ini file for the user to save personal information and to automatically read when booting the software next time.

The interface display module is mainly to display each control, including window, fix, button, table, lable and so on. The layout of the control has a certain hierarchy, and finally the whole interface is formed. The entire interface is attached to a window so that it is necessary to first of all generate a window

The log-in module mainly implements the communication between the server and the RDP protocol. The concrete realization function is void log-in (GtkWidget *widget, gpointer data); it is called for use when users click on the log-in button. Firstly, it accesses to the domain control address, user name, password, IP address and other information input by the users.

The command interaction module mainly implements the instruction interaction with the server. The user sends msg command messages to the server, the server returns msg_response to the user, and then the user extracts the valid information.

\section{Conclusion}

This paper takes the combination of wireless sensor networks and cloud computing as a starting point and integrates the advantages of both. The ZigBee wireless transmission protocol that is currently widely used is applied to build wireless sensor network. And the embedded ARM-Linux system real-time performance and high reliability are adopted to complete the data processing and transfer work. Finally, the remote access is completed equipped with virtual desktop cloud system. This design uses the CC2430 chip of TI company to complete the hardware design of the wireless module, and completes the cutting and software application work based on the ZigBee2006 protocol stack. At the same time, the S3C2440 chip is selected as the core control chip to complete the porting of embedded Linux and serial port and communication work. And based on the Microsoft remote desktop protocol, the development of the virtual cloud desktop client is completed in the Linux environment so that it can remotely access to the server resources. 


\section{References}

[1] He, J., \& Chen, Y. (2016). Remote monitoring system of an agricultural tillage machine based on an embedded arm technology wireless sensor. International Journal of Online Engineering, 12(5), 33. https://doi.org/10.3991/ijoe.v12i05.5732

[2] Biswas, J., \& Bera, P. (2016). Pi-based load frequency controller design for multi machine system using genetic algorithm. 65.

[3] Li, Y., Xu, M., \& Tian, S. (2016). Grading system design of dendrobium officinale based on machine vision., 14(3A), 217. https://doi.org/10.12928/telkomnika.v14i3a.4396.

[4] Arm, J., Bradac, Z., \& Kaczmarczyk, V. (2016). Real-time capabilities of linux rtai. IFACPapersOnLine, 49(25), 401-406. https://doi.org/10.1016/j.ifacol.2016.12.080

[5] Rahman, I., Hollitt, C., \& Zhang, M. (2016). Contextual-based top-down saliency feature weighting for target detection. Machine Vision \& Applications, 27(6), 893-914. https://doi.org/10.1007/s00138-016-0754-x

[6] Kobayashi, T. (2016). Discriminative local binary pattern. Machine Vision \& Applications, 1-12. https://doi.org/10.1007/s00138-016-0780-8.

\section{Authors}

Chunqing Han and Lin Li are with Binzhou Tobacco Monopoly Bureau, Shandong, China (hanchunqing0807@163.com).

Article submitted 25 August 2017. Published as resubmitted by the authors 05 October 2017. 